\title{
Super Environmental Adaptability of SARS CoV2
}

\author{
Zebunnesa Zeba
}

\begin{abstract}
Assistant Professor, Department of Public Health and Informatics, Jahangirnagar University, Savar, Dhaka-1342, Bangladesh, Email:zebunnesa.zeba@juniv.edu; zeba5533@gmail.com, Phone: +8801711106654
\end{abstract}

The pandemic diseases have been some of the most distressing happenings in the history of mankind ${ }^{1}$. At present, the world is facing the Covid-19 pandemic. It is caused by the severe acute respiratory syndrome-coronavirus (SARS-CoV-2), which is a single-stranded positive-sense RNA virus and member of the Coronaviridae family, identified in the mid-1960s that causes infection and respiratory failure ${ }^{2,3}$, and has led to the thousands of people deaths ${ }^{4,5}$. In 2019 , it was first discovered in Wuhan city, China ${ }^{6}$. This virus has emerged from China during cold weather when the temperature was near zero degree Celsius. Though many viruses cannot survive at this lower temperature, the SARS $\mathrm{CoV} 2$ has that ability. It spread from China with a cold temperate country all over the world. A large number of cases of corona-affected people have also been found from high temperate regions in the Middle East. Therefore, these indicate that SARS CoV2 has an enormous ability to survive in any kind of temperature. In Brazil also found that there was no evidence supporting that case counts of COVID-19 could decline when the weather becomes warmer, in temperatures is above $25.8^{\circ} \mathrm{C}^{7}$.

The relationship between the prevalence of the Covid-19 with maximum air humidity, and wind speed was negligible and statistically not significant. In addition, with increasing temperature, the prevalence of the disease has decreased $^{8}$. The average temperature and daylight hours have shown a positive association towards the spread rate of COVID-19 ${ }^{1}$. The growth rate of COVID-19 cases peaked at a temperature of $\sim 5^{\circ} \mathrm{C}$ and a humidity of 0.6 to $1 \mathrm{kPa}$ in the temperate regions of the Northern Hemisphere during the outbreak month, while it decreased in regions that had warmer or colder temperatures9. Likewise, average pressure, average temperature, minimum temperature, and average water vapor pressure were found to be significantly correlated with the incidence of COVID-19 $9^{10,11}$. Generally, the largest contiguous syndromes outbreak in the regions of cooler weather, leading to the theories that high temperatures might begin to the tail of in the summer season ${ }^{1}$. Similarly, in the early outbreak of Covid-19 in Bangladesh, it was perceived by people that, this virus would not stay in there due to the temperate zone. However, later, it was proved as a wrong concept. Moreover, that false perception influenced negatively among decision and policymakers in Bangladesh; as well as, it lengthened the strong emergency preparedness against the spread and control of the virus. As a result, the number of corona-affected people has been increasing day by day, in spite of persisting hot temperatures.

This ability of a virus to survive in the high and low temperatures is also a potent virulent factor. In the case of other viruses, this type of ability is very rare. It has a wide range of temperatures to grow. It has been reported that this virus can survive in the metal and other objects in a significant duration of time; which is also a virulent factor for the virus. However, this pattern of survival ability of the coronavirus in the environment is not seen so far. Scientists are searching the clues regarding this potency of the virus. It has been postulated that SARS CoV2 can mutate its gene according to the environmental factors. The rapidly evolving nature by changing the host body environment and extreme environmental stability collectively makes SARS CoV-2 into an extremely virulent genetic variant. The evolution of the virus has been occurred by the continuous process of molecular genetic manipulation, such as, through mutation, deletion, and genetic recombination events ${ }^{12}$. The 
genetic sequencing is also performed by BCSIR (Bangladesh Council of Scientific and Industrial Research) and NILMRC (National Institute of Laboratory Medicine \& Referral Centre) where several mutations have been detected. This may be the reason for the survival ability of the virus in any environment. Thus, the potency and virulence of the virus are increasing day by day. Since the SARS $\mathrm{CoV} 2$ virus is a new pathogen, research is still needed to find out the cause of its adaptability with different environments and climate.

Most of the Virus of human diseases cannot survive in an open environment. It is an obligate intracellular pathogen. SARS CoV2 cannot grow without human cell. Artificial culture of the virus in the lab has been first done in Australia, and later by other several countries. The in vitro viral culture has needed a range of 35 to 37 degrees Celsius, which is the core temperature of the human body. However, the effect of environmental factors such as ambient temperature, humidity, relating to the COVID-19 pandemic has not been sufficiently investigated $^{4}$. Dealing with COVID-19 and preventing its rapid and dangerous spread is a global challenge. However, due to the potential variability of this disease, according to the type of climate and other environmental factors, its prevention and control should be investigated quickly and seriously to protect public health ${ }^{13}$. Though, the rapid development of 2019-nCoV vaccine, it still faces many unknown challenges, including the antigenic characteristics of the 2019$\mathrm{nCoV}$, the influence of antigenic variation, the protective immune response of host, the protection of the elderly population, and the downstream manufacturing process of the new vaccine. The safety and efficacy of vaccines are the first priority for vaccine development and should be carefully evaluated.

\section{Reference}

1. Iqbal MM, Abid I, Hussain S, Shahzad N, Waqas MS,
Iqbal MJ. The effects of regional climatic condition on the spread of COVID-19 at global scale. Sci Total Environ. 2020;739:140101.

2. Swerdlow DL, Finelli L. Preparation for Possible Sustained Transmission of 2019 Novel Coronavirus. JAMA. 2020;323(12):1129-30.

3. Wu F, Zhao S, Yu B, Chen YM, Wang W, Song ZG, et al. A new coronavirus associated with human respiratory disease in China. Nature. 2020;579(7798):265-9.

4. Eslami H, Jalal M. The role of environmental factors to transmission of SARS-CoV-2 (COVID-19). AMB Express. 2020;10(1)

5. Thienemann F, Pinto F, Grobbee DE, Boehm M, Bazargani N, Ge J, et al. World Heart Federation Briefing on Prevention: Coronavirus Disease 2019 (COVID-19) in LowIncome Countries. Glob Heart. 2020;15(1).

6. Lu H, Stratton CW, Tang Y. Outbreak of pneumonia of unknown etiology in Wuhan, China: The mystery and the miracle. J Med Virol. 2020;92(4):401-2.

7. Prata DN, Rodrigues W, Bermejo PH. Temperature significantly changes COVID-19 transmission in (sub) tropical cities of Brazil. Sci Total Environ. 2020;729:138862.

8. Bhattacharjee S. Statistical investigation of relationship between spread of coronavirus disease (COVID-19) and environmental factors based on study of four mostly affected places of China and five mostly affected places of Italy. arXiv [Internet]. 2020; Available from: https://arxiv.org/abs/2003.11277

9. Ficetola GF, Rubolini D. Climate affects global patterns of COVID-19 early outbreak dynamics. Medrxiv. 2020;

10. Aboubakr HA, Sharafeldin TA, Goyal SM. Stability of SARS-CoV-2 and other coronaviruses in the environment and on common touch surfaces and the influence of climatic conditions: A review. Transbound Emerg Dis. 2020;

11. Li J, Zhang L, Ren Z, Xing C, Qiao P, Chang B. Meteorological factors correlate with transmission of 2019nCoV: Proof of incidence of novel coronavirus pneumonia in Hubei Province, China. Medrxiv. 2020;

12. Das P, Choudhuri T. Decoding the global outbreak of COVID-19: the nature is behind the scene. VirusDisease. 2020;31(2):106-12.

13. Askari SG, Khatbasreh M, Ehrampoush MH, Sheikhha $\mathrm{MH}$, Eslami H, Taghavi M, et al. The relationship between environmental exposures and hormonal abnormalities in pregnant women: An epidemiological study in Yazd, Iran. Women and Birth. 2018;31(3):e204-9

[Bangladesh Journal of Infectious Diseases, October 2020;7(suppl_2):S1-S2]

How to cite this article: Zeba Z. Super Environmental Adaptability of SARS CoV2. Bangladesh J Infect Dis 2020;7(suppl_2):S1-S2 